\title{
Viral Infection: An Evolving Insight into the Signal Transduction Pathways Responsible for the Innate Immune Response
}

\author{
Girish J. Kotwal, ${ }^{1}$ Steven Hatch, ${ }^{2}$ and William L. Marshall ${ }^{2}$ \\ ${ }^{1}$ University of Medicine and Health Sciences, St. Kitts, New York, NY 10001, USA \\ ${ }^{2}$ Division of Infectious Disease and Immunology, Department of Medicine, University of Massachusetts Medical School, \\ 364 Plantation Street, Worcester, MA 01605, USA
}

Correspondence should be addressed to William L. Marshall, william.marshall@umassmed.edu

Received 7 February 2012; Accepted 21 June 2012

Academic Editor: Julia G. Prado

Copyright (C) 2012 Girish J. Kotwal et al. This is an open access article distributed under the Creative Commons Attribution License, which permits unrestricted use, distribution, and reproduction in any medium, provided the original work is properly cited.

The innate immune response is initiated by the interaction of stereotypical pathogen components with genetically conserved receptors for extracytosolic pathogen-associated molecular patterns (PAMPs) or intracytosolic nucleic acids. In multicellular organisms, this interaction typically clusters signal transduction molecules and leads to their activations, thereby initiating signals that activate innate immune effector mechanisms to protect the host. In some cases programmed cell death-a fundamental form of innate immunity-is initiated in response to genotoxic or biochemical stress that is associated with viral infection. In this paper we will summarize innate immune mechanisms that are relevant to viral pathogenesis and outline the continuing evolution of viral mechanisms that suppress the innate immunity in mammalian hosts. These mechanisms of viral innate immune evasion provide significant insight into the pathways of the antiviral innate immune response of many organisms. Examples of relevant mammalian innate immune defenses host defenses include signaling to interferon and cytokine response pathways as well as signaling to the inflammasome. Understanding which viral innate immune evasion mechanisms are linked to pathogenesis may translate into therapies and vaccines that are truly effective in eliminating the morbidity and mortality associated with viral infections in individuals.

\section{Introduction}

The innate immune system is as ancient as the bacterial immune response to bacteriophages. As the nature and complexity of viral innate immune evasion mechanisms evolved, so has the innate-and eventually adaptiveimmune response to these mechanisms. The innate immune response in mammals is initiated by the interaction of stereotypical pathogen components with germ-line encoded receptors. In some cases, signal transduction pathways are stimulated in sentinel cells, such as macrophages and dendritic cells. Stimulation of these signaling pathways promptly activates innate effector mechanisms to protect the host; these innate immune signals also activate antigen-presenting cells that are critical to the eventual adaptive immune response of the host [1]. In this paper we will summarize findings in the innate immune system that are relevant to viral pathogenesis and outline the evolution of viral mechanisms that suppress innate immunity in mammalian hosts.

\section{The Innate Immune System}

The receptors of the innate immune system are germline encoded and include the nucleotide-binding domain leucine-rich repeat containing receptors, the Toll-like receptors (TLRs), and the RIG-I-like receptors (RLRs). The RLRs are cytosolic sensors of pathogen RNA and include proteins encoded by the retinoic acid-inducible gene-I (RIG-I) [2], the melanoma differentiation-associated gene 5 (MDA5) $[3,4]$, and the laboratory of genetics protein 2 (LGP2) [4] and DDX3, which is thought to associate with RIGI [5]. The helicase domains of RLRs detect the cytosolic RNA of microbial pathogens, generating signals that drive 
production of cytokines and interferons. Helicases are ATPdependent enzymes that unidirectionally translocate along a transcript thereby dissociating nucleic acid duplexes [6]. The RIG-I and MDA5 RLRs play critical roles in the recognition of foreign RNA and in the response to many viral pathogens. MDA5 and RIG-I contain a DExD/H-box RNA helicase domain and caspase activation and recruitment domains (CARDs). RIG-I recognizes $5^{\prime}$-triphosphate RNA, and MDA5 can recognize complex webs of pathogen RNA, comprised of both viral single-stranded and double-stranded RNA [2]. The LGP2 RLR protein was found to lack a CARD domain and was originally identified as a dominant negative inhibitor of RIG-I signaling [7]. Under some circumstances, though, it appears LGP2 can stimulate RLRs such as MDA5 and RIG-I [8]. CARD engagement leads to interaction with a protein known as mitochondrial antiviral signaling protein (MAVS) that is alternatively designated CARDIF, HELICARD, or IPS-1 (referred to here as MAVS) $[9,10]$. Subsequently, upon oligomerization, MAVS signals to members of the IKK family of kinases that are critical for the innate immune response [10]. Thus MAVS induces IKK $\alpha$ and IKK $\beta$ stimulation that leads to translocation of NF- $\kappa \mathrm{B}$, as well as IKKe/TBK1 stimulation that leads to translocation of IRF-3. These transcription factors stimulate production of cytokines, other innate immune response proteins, and type I interferons [4].

Extracytosolic innate immune sensing of pathogens is mediated via the TLRs. Humans are known to encode ten TLRs which are each involved in the recognition of different pathogen-associated molecular constituents [11]. The TLRs are transmembrane receptors found on the cell surface and/or associated with endocytic vesicles [11]. Thus, they are ideally situated to detect extracytosolic pathogens. For example, TLR4 is required for the recognition of Gram-negative bacterial lipopolysaccharide (LPS, or endotoxin) while TLR3 is able to recognize dsRNA, a signature compound common in the lifecycle of many viruses, while TLR7 and 8 recognize ssRNA [12]. Toll/IL-1 interacting receptor (TIR) adapters ultimately stimulate IkB family kinases (IKKs) often via transducing proteins such as IRAKs and TRAFs, thereby mediating signaling originally induced by engagement of TLRs that ultimately activates NF- $\kappa$ B and IRF3 [13].

Two classical IKKs, IKK $\alpha$ and IKK $\beta$, are critical for NF- $\kappa \mathrm{B}$ activation. They function, in large part, by phosphorylating the inhibitors of NF- $\kappa \mathrm{B}$, known as IkBs. Once phosphorylated, IkBs are ubiquitinated and degraded. This allows NF- $\kappa \mathrm{B}$ subunits to translocate to the nucleus and activate target gene expression. NF- $\kappa \mathrm{B}$ is critical for driving the expression of numerous cytokines, chemokines, and costimulatory molecules, creating an inflammatory response [14]. On the other hand, the two nonclassical IKK family members, IKK $\varepsilon$ and TBK1, are implicated in IRF3 activation. In particular, they are believed to directly phosphorylate several serine residues within the C-terminal activation domain of IRF3. Once phosphorylated, IRF3 dimerizes and translocates to the nucleus where it activates target gene expression [15]. IRF3 activation is critically important for the activation of type I interferons, either directly [16] or via an autocrine/paracrine loop $[17,18]$. Type I interferons, in turn, are capable of inducing a significant antiviral response in the host $[2,13,19]$.

Cells also encode cytosolic DNA sensors which detect DNA, which is not typically present in the cytosol and thus a pattern whose recognition signals the presence of viral nucleic acids. Nucleotide oligomerization domain-like receptor proteins (NLRs) are implicated in the intracytosolic recognition of sterile inflammatory instigators, such as urate crystals, intracytosolic DNA, or viral RNA. One such NLR, nucleotide oligomerization domain-like receptor protein 3 (NLRP3) is an inflammasome component that signals to the apoptosis-associated speck-like protein containing a caspase recruitment domain (ASC) to induce the clustering-induced self-processing of procaspase 1 into caspase 1 which then digests the precursor form of pro-IL-1 $\beta$ and pro-IL-18 to permit release of the inflammatory cytokines IL- $1 \beta$ and IL-18 from the cell [20]. Similar inflammatory pathways are triggered by engagement of other cytosolic DNA sensors such as AIM-2, another NLR that ultimately induces cleavage of procaspase 1 into caspase 1 . Aim-2 can detect the molecular patterns of intracellular hazards such as pathogen DNA, particularly that of poxviruses [21]. Other NOD proteins which are alternatively designated NACHT, LRR, and PYD function as sensors of toxic intracellular molecules including cytosolic DNA [22]. Thus the NLRs represent examples of cytosolic DNA sensors capable of inducing an inflammatory antiviral response.

Another antiviral cytosolic sensor is the DNA-dependent activator of interferon (DAI), which binds B- and Z-form DNA, thereby recognizing intracytosolic viral DNA. Signals from such sensors are transduced by known innate immune kinases such as TBK1, which interacts with a protein known as stimulator of interferon genes (STINGs) to activate NF$\kappa \mathrm{B}$ and IRF3 signaling $[10,23,24]$. Finally, it is assumed that there is at least one other pathway for the detection of the dsDNA of microbes, based in part on DNA sensing in cells despite absence of the DAI pathway [25]. The known receptors for viral DNA ultimately induce interferons, cytokines, and programmed cell death pathways.

Apoptosis is the programmed death of dangerous or unnecessary cells, for example, virally infected, aging, or malignant cells. It is thus one of the most ancient forms of innate immunity. Certain cellular bcl-2 proteins mediate resistance to programmed cell death (apoptosis) [50-52], typically via interaction with proapoptotic bcl-2-related proteins [52]. Human bcl-2 also leads to increased nuclear translocation of the transcription factor, NF- $\kappa \mathrm{B}$ [53-55], which typically promotes cell survival $[14,56,57]$. Other cellular bcl-2 proteins promote cell death in response to harmful stimuli such as viral infection. Other effectors of programmed cell death are caspases. Cleavage of cellular caspases and/or loss of mitochondrial integrity promote cell death in the face of many stimuli including viral infection. Still other death programs include pyroptosis-the death of cells following activation of the PYRIN domains and IL-1 release. Thus, in the absence of viral innate immune evasion, apoptosis provides an antiviral mechanism for the elimination of virally infected cells. 
TABLE 1: Examples of parallel evolution of viral innate immune evasion mechanisms.

(a) Bacteria

\begin{tabular}{lll}
\hline Mechanism & Viral evasion strategy & Virus protein \\
\hline CRISPER & Genetic variation of DNA & DNA polymerase [26] \\
Psp-induced signaling & Unknown (Psp genetic variation) & Unknown \\
Restriction/methylation & Methylation of viral target DNA & Acquired bacterial methylase \\
Apoptosis & Lysogeny/tolerance & [26] Phage lysis gene regulation [27] \\
\hline
\end{tabular}

(b) Drosophila

\begin{tabular}{lll}
\hline Mechanism & Evasion strategy & Viral protein \\
\hline DICER1 & Genetic variation of DNA & Virus encodes miRNAs [28] \\
Vago & $\mathrm{IkB}$ Jak/Stat inhibitors & Viral IkB, unknown inhibitor of Jak/STAT [29] \\
DICER2 & Binding of ds RNA & FHV-B2 protein [30] \\
Apoptosis & Inhibitor of apoptosis (IAP) & Baculovirus p35 [31, 32] \\
\hline
\end{tabular}

(c) Mammalian systems

\begin{tabular}{lll}
\hline Mechanism & Evasion strategy & Viral protein \\
\hline & dsRNA binding & Influenza virus NS1 [33], VACV E3 (also \\
inhibits DNApol III DNA sensing) [34]
\end{tabular}

Interferon signaling

Inhibition of signaling

ibition of IFN binding

Inhibitors of RIG-I

Inhibitors of MDA5, LGP2

Inhibitors of DDX3

Viral RNA/DNA sensing

\begin{tabular}{|c|c|}
\hline & $\begin{array}{l}\text { Inhibitors of the AIM } 2 / \mathrm{N} \\
\text { Inflammasome }\end{array}$ \\
\hline & $\begin{array}{l}\text { Inhibitors of proteins acti } \\
\text { downstream of the AIM2 } \\
\text { inflammasome }\end{array}$ \\
\hline & DAI \\
\hline & Viral inhibitor of apoptos \\
\hline Programmed cell death & Viral bcl-2s \\
\hline & Blockade of IL-1-mediate \\
\hline $\begin{array}{l}\text { Evolution of the } \\
\text { Innate Immune } \\
\text { Recurring Them }\end{array}$ & Different Genes, \\
\hline
\end{tabular}

Prokaryotic organisms encode primordial proteins that recognize the molecular patterns (e.g., specific sequences of DNA of bacteriophages) from pathogens (i.e., bacteriophages) and thus can be considered to possess a primitive innate immune system. Although the mechanisms of innate immunity in bacteria differ radically from those of higher organism, four principles of innate immunity are preserved in several mechanisms (Table 1). First, the clustered regularly space short palindromic repeats (CRISPERs) of bacteria and archaea encode a series of palindromic sequences that target pathogen DNA and suppress their transcription in a way similar to the antiviral action of microRNAs of Drosophila $[58,59]$. Second, following exposure of prokaryotes to bacteriophages, the phage shock protein (Psp) signaling pathway 
involves an unknown sensor and signal transduction by the leucine zipper protein PspB. PsP signaling is initiated in response to loss of cell membrane integrity induced by stresses such a bacteriophage infection [60]. This is similar in principle to the enhanced cell membrane integrity mediated by interferon in the mammalian antiviral response $[2,13,19]$. A third conserved principle is intracytosolic nucleic acid recognition (analogous to mammalian RLRs or Drosophila DICER) that triggers an innate immune response. In bacteria, restriction endonucleases recognize and digest bacteriophage nucleic acids, while specificity of this response is maintained by bacterial methylation of its native DNA. Fourth, programmed death of bacteriophage-infected bacteria induced by the MazF protein can pre-empt spread of viral infection, as is true of proapoptotic proteins in higher organisms (reviewed in [27]). Bacteriophage mechanisms to evade the bacterial innate immune pathways include rapid mutation to generate DNA sequence diversity that evades CRISPER, acquisition of host bacterial methylases to mask restriction sites in bacteriophage DNA [26], and programmed cell death resistance [27]. A bacteriophage mechanism to evade PsP signaling has not been reported, although it is tempting to speculate that the rapid mutation observed during bacteriophage infection might avoid detection by the Psp pathway. Although the details and evolution of innate immune mechanisms in bacterial cells are highly divergent from multicellular organisms (Table 1), the principal functional attributes of innate immune recognition and viral evasion are remarkably conserved, especially in the invertebrate innate immune responses.

Drosophila lack an adaptive immune system; thus, they are ideal model organisms to study innate immunity since they possess a complex innate immune system (Table 1). Viral mechanisms for suppression of innate immunity in Drosophila have been reviewed recently and will be discussed only briefly here [29]. In contrast to mammalian cells, Drosophila does not encode NOD proteins. It has been suggested that Drosophila TLRs encoded recognize PAMPs of viruses that are tropic for Drosophila [61]. Unlike mammals, Drosophila rely heavily on RNA interference as a defense against viruses. The protein DICER2 is a helicase/endonuclease that is related to the RIG-I-like helicase of mammals [62]. DICER2 has two effector functions; the first initiates a cascade of endonucleolytic cleavage of viral RNAs that mediate gene silencing, and the second is a RIG-Ilike signaling activity of DICER2 whereby DICER-2 mediates induction of the antiviral genes, such as Vago [62]. A distinct protein, DICER1, cleaves isolated miRNAs that subsequently suppress transcription of viral RNA just as mammalian DICER2 does $[58,59]$. To evade this innate immune defense, the Flockhouse virus encodes the dsRNA-binding protein B2 that inhibits dsRNA recognition by DICER1 and DICER2 in Drosophila [30]. Furthermore, viruses inhibit the function of inhibitors of kappaB (I- $\kappa \mathrm{B})$ translocation to prevent signaling initiated by Drosophila TLRs [61], but not the Jak/Stat antiviral defense pathway in Drosophila. Other viral proteins that act in signal transduction are thought to mediate the production of antiviral peptides, including the principal Drosophila gene induced by viral infection that is Vago, which encodes a 14 kilodalton cysteine-rich polypeptide [62]. Vago is thought to be, in principle, analogous to interferons as it is a virus-induced protein critical to control viral infection. Finally, viral innate immune evasion proteins encoded by baculovirus inhibit the function of apoptotic pathways $[31,32,63,64]$. Although there are parallels between the principles of innate immune defense against viruses between bacteria and Drosophila (Table 1), the innate immune responses of Drosophila more closely resemble those of the mammalian antiviral innate immune response.

\section{Viral Evasion of Host Defenses: Highlighting Critical Components of the Mammalian Innate Immune Response}

4.1. DNA Viruses. The Poxviridae are large enveloped DNA viruses that replicate in the cytoplasm. Vaccinia virus (VACV) is a robust poxviral vaccine originally used to eradicate smallpox. Poxviruses encode approximately 180 genes. About 80 genes are essential for replication in tissue culture, whereas 100 encode virulence proteins, such as decoy receptors for IL-1, TNF- $\alpha$, and interferons. These virulence proteins (Table 1, bold text, and Figure 1) interdict innate immune signaling by preventing receptor engagement at the cell surface [65]. Moreover, the pox virus proteins, E3 and $\mathrm{K} 3$, bind dsRNA in the cytoplasm, reducing type I interferon production and, in the case of $\mathbf{E} 3$, preventing activation of the dsRNA-dependent protein kinase PKR [34].

Other vaccinia virus proteins have been characterized as inhibitors of innate immune intracellular signal transduction (Figure 1). For example the VACV N1 family of ten bcl-2 like proteins inhibits NF- $\kappa$ B signaling [37]. Of the proteins characterized to date, $\mathrm{N} 1$ is the most robust VACV virulence factor, increasing replication 10,000-fold, inhibiting NF- $\kappa$ B, IRF3 and apoptotic signaling [35, 66, 67]. A52 inhibits NF- $\kappa \mathrm{B}$ and increases p38 kinase activity [68]. A46 inhibits $\mathrm{NF}-\kappa \mathrm{B}$ and IRF3 signaling [36]. And K7 inhibits IRF3 and $\mathrm{NF}-\kappa \mathrm{B}$ signaling by binding to DDX3 and preventing MAVS signaling to TBK1 [5]. It is unclear what the role of N1's antiapoptotic function is as VACV already encodes a vbcl-2 (F1), that, unlike $\mathrm{N} 1$, is critical for viral survival in vitro. Thus, the antiapoptotic potential of the $\mathrm{N} 1 \mathrm{vbcl}-2$ reconciles the absence of cell death despite N1 inhibition of NF- $\kappa$ B. While no direct inhibitor of the inflammasome has been detected in vaccinia virus, the poxvirus serpins SPI-1 and crmA inhibit caspase 1 activity downstream of the inflammasome [69] and another poxvirus, myxoma virus encodes the M013 PYRIN domain containing protein that inhibits signaling by the inflammasome by interrupting association of NLRP3 and ASC ([45], see Figure 2). Thus poxviruses inhibit many aspects of the two-signal inflammasome inflammatory pathway by inhibiting pro-IL-1 $\beta$ and pro-IL- 18 production by the IKK pathway and cleavage by the inflammasome (Figure 2). Additionally, as outlined in Figure 1, IKK complex signaling to TNF- $\alpha$, IFN, and other cytokines is impaired by viral innate immune evasion proteins.

The gammaherpesviruses encode proteins that highlight the role of antiapoptotic factors in innate immune evasion. 


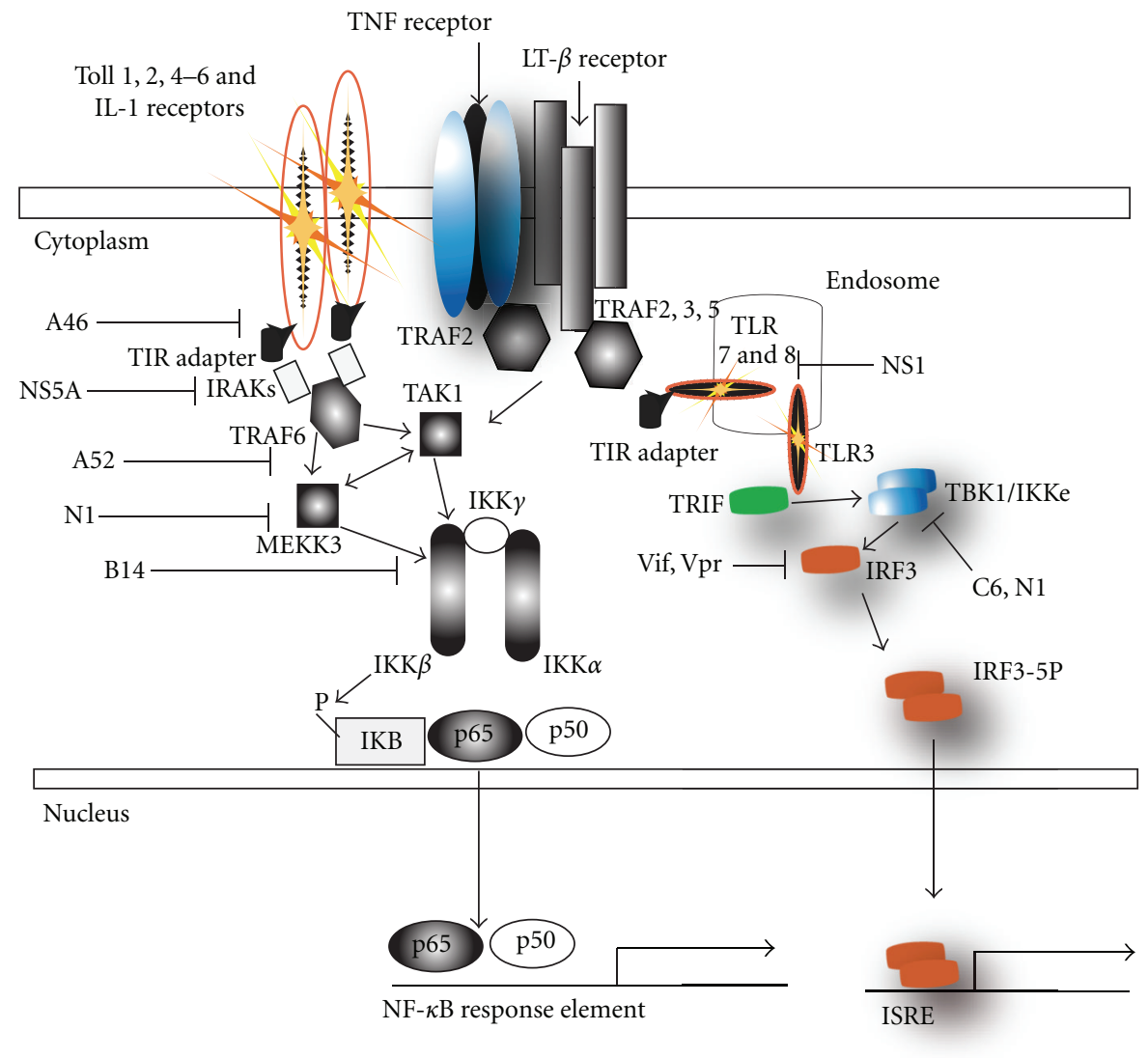

FIgURE 1: Signaling by the innate immune system that is inhibited by several viral proteins. This model depicts the salient features of TLR-induced NF- $\kappa$ B and IRF3 induction. Several poxviral proteins N1, C6, A14 A46, and A52 inhibit the activation of NF- $\kappa$ B and/or IRF3 signaling pathways, by interacting with and inhibiting the activity of the classical IKK complex (IKK $\alpha / \beta / \gamma)$ as well as the nonclassical IKKe/TBK1 complex. HCV protein NS5A inhibits TIR signaling by MyD88, its NS3/4A digests MAVS to inhibit RLR signaling, and its core protein inhibits Jak/Stat signaling. Finally, HIV Vif and Vpr degrade IRF3.

Herpesviruses cause a latent, life-long infection. During latent infection herpesvirus antigens are principally present in the nucleus thereby evading recognition by the cytosolic and extranuclear membrane-associated components of the innate immune system. The apoptotic mechanisms of the innate immune system affect the elimination of herpesvirus-infected cells. Two human gammaherpesviruses, Epstein Barr Virus (EBV) and Kaposi's Sarcoma Herpesvirus (KSHV) have evolved several mechanisms that induce latent infection and thus inhibit the apoptotic innate immune response (LMP1 and LANA1). Recent studies of recombinant EBV containing deletions of the genes encoding both the antiapoptotic vbcl-2, BHRF1 [47], and a second EBV bcl-2, BALF1 $[48,70,71]$, have revealed that deletion of both EBV bcl-2 homologs dramatically increases the survival of cells undergoing EBV infection [72]. Finally, the KSHV gammaherpes orf63 has been shown to encode a viral NLR (vNLR, Figure 2) that inhibits NLRP1, NLRP3, and NOD2 function, permitting persistent KSHV infection [45].

Viral caspase inhibitors are believed to neutralize immune responses of the host that activate the caspase pathway of apoptotic cell death (although it is logical to hypothesize that viral caspase 1 inhibitors also inhibit the inflammasome). Three different viral proteins inhibit the caspase pathway of apoptosis: (1) the serpins of the poxviruses, exemplified by crmA, a caspase inhibitor encoded by the cowpox virus genome [69], and (2) the baculovirus p35 caspase inhibitor protein [73]. Finally, the v-FLIPs, which are expressed by the gammaherpesviruses equine herpesvirus 4 and Kaposi's Sarcoma Herpesvirus (KSHV/HHV-8) [46] inhibit apoptosis by competing with caspase 8 (FLICE) for binding to the death effector domains of adaptor proteins of death receptors (reviewed by [74]). Inhibition of the activation of the caspase cascade that would otherwise follow oligomerization of death receptors prevents apoptosis induced by the cascade of proteases that eventually induce cellular self-digestion [75]. Inhibition of caspases is thus another evolutionarily conserved mechanism for viruses to avoid the apoptotic host innate immune response. In addition, vFLIPs dysregulate the function of IKK- $\gamma$, thereby activating the IKK complex-mediated dissociation of IkB from NF- $\kappa \mathrm{B}$ and subsequent NF- $\kappa \mathrm{B}$ signaling [76]. Activation of the IKK cascade that would otherwise promote innate immune signaling activates KSHV replication and promotes KSHV-transformed cell survival. In the case of this KSHV mechanism, signaling by the classical innate immune 


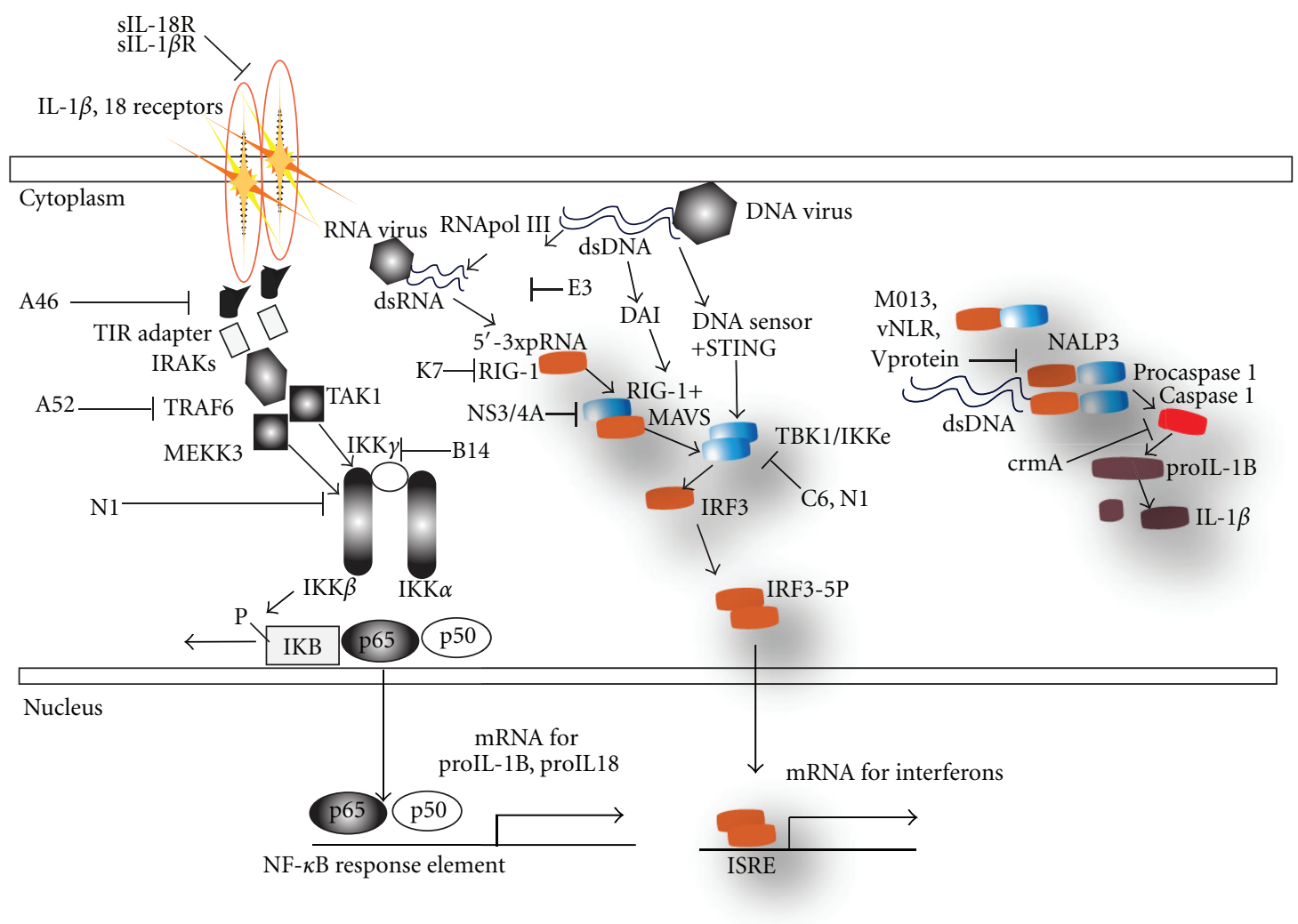

FIGURE 2: Viral proteins inhibit nucleic acid receptors of the intracytosolic innate immune response. Viruses inhibit each of the two signals that initiate the inflammasome activation process. The first signal-IL-1 $\beta$ and/or IL-18 binding and activation of the TLR/IL- $1 \beta$ receptor pathway-is inhibited by soluble IL- $1 \beta$ and IL-18 (from VACV); downstream, inhibitors of signaling to NF- $\kappa$ B (from VACV or HCV) repeatedly target this important antiviral pathway that optimally requires NF- $\kappa$ B translocation leading to the production of pro-IL- $1 \beta$ and pro-IL-18. Second, the inflammasome processes these pro-IL-1 $\beta$ and pro-IL-18 proteins via caspase- 1 that is itself processed upon clustering mediated at the NLRP3 inflammasome upon detection of intracytosolic pathogens. This leads to IL- $1 \beta$ and IL-18 production and release that activates the IL-1 $\beta / \mathrm{IL}-18$ pathway in an autocrine manner, as well as the innate and adaptive immune response. Inflammasome activation is inhibited by myxoma virus M013, measles viruses V protein, and KSHV vNLR. Finally signaling to IRF3 by intracytosolic DNA or RNA is inhibited at the level of MAVS by HCV's NS3/4A and at the level of TBK1 by VACV C6 and N1 (Figure 1).

response pathway is perturbed by KSHV protein, allowing KSHV to escape the apoptotic host innate immune response.

4.2. RNA Viruses. Hepatitis C Virus (HCV) is a singlestranded, positive-sense RNA virus that encodes 10 proteins. HCV typically induces a life-long infection via successfully evading the adaptive and innate immune responses. The many HCV proteins that possess dual functions in both replication and innate immune evasion likely reflect the limited number of HCV. Surprisingly for the small size of its genome, HCV shares mechanisms of innate immune evasion with much larger DNA viruses such as the poxviruses. Poxvirus nucleoside triphosphate phosphohydrolase I (NPH-I) is absolutely essential for VACV mRNA transcription and VACV replication, yet simultaneously NPH-I inhibits the interferon response [77]. The HCV NS3/4A protease/helicase encodes a helicase that suppresses the IFN- $\beta$ promoter independently of NS3/4A proteolytic destruction of innate immune signaling components such as TRIF that activate the IFN- $\beta$ [42]. A recombinant RLR engineered to encode only a helicase domain is a dominant negative inhibitor of RLR-driven interferon (IFN)- $\beta$ promoter activity. This dominant negative RLR lacks a signaling domain [7]. Viral helicases inherently lack signaling domains, and thus viral helicases structurally resemble a dominant negative RLR and might act as RLR antagonists. HCV innate immune evasion mechanisms also include the proteolytic destruction of MAVS by the protease component of the NS3/4A protein. The destruction of MAVS, which transduces signals from the RLRs, therefore inhibits signaling to IRF3 via TBK1, blocking the interferon response. HCV core protein expression correlates with impaired signaling of the Jak/Stat pathway to IFN$\alpha / \beta$, although the mechanism for this is still being defined [78]. The HCV core protein inhibits TLR signaling through its chronic stimulation of TLR2 resulting in TLR hyporesponsiveness [79], HCV core protein binds STAT1, and HCV infection leads to STAT1 degradation, which inhibits the antiviral signaling in the Jak/Stat pathway [80]. Finally HCV protein NS5A inhibits recruitment of IRAK to the MyD88 
TIR adapter [40] and may inhibit interferon production via NS5A suppression and the phosphorylation of eIF2 by the PKR kinase [40]. Thus, HCV inhibits several types of innate immune signaling via the action of only a few proteins.

Influenza viruses are enveloped RNA viruses with a negative-sense, single-stranded segmented genome. Influenza virus is an extremely virulent respiratory pathogen, and influenza virus possesses distinct innate immune evasion mechanisms that are critical for its pathogenesis. The nonstructural protein-1 (NS1) functions to inhibit the host interferon response [81], thus inhibiting activation of IRF3 [35, 81]. Deletion NS1 dramatically attenuates influenza viruses [35, 81]. Inhibition of RLR signaling is a critical event in the lifecycle of many viral pathogens, for example, influenza virus $[82,83]$. Influenza NS1 protein inhibits RIGI signaling to NF- $\kappa$ B and IRF3 by inhibiting the E3 ubiquitin ligase TRIM 25 required for its function [33]. Furthermore, influenza virus NS1 binds to dsRNA that would trigger the RIG-I/MDA5 antiviral response. Finally, the influenza virus polymerase activity depends upon cellular mRNA, thereby depleting host mRNAs, which has been postulated to inhibit host antiviral gene expression [45]. Similar to herpesviruses, influenza virus undergoes replication in the nucleus minimizing detection by intracytosolic nucleic acid sensors dsRNA binding by the influenza. Thus, influenza virus appears to encode inhibitors of nucleic acid sensing, host antiviral gene expression, interferon response, dsRNA, and physical separation of signal transduction components from the innate immune sensors. These mechanisms are a recurrent theme in viral innate immune evasion.

Retroviruses are highly successful at evading innate and adaptive immune responses. The rapid evolution of HIV envelope proteins and their heavy glycosylation results in epitopes that are not conducive to an adaptive immune response. The well-studied mechanisms for retroviral immune evasion include the infection and apoptotic destruction of HIV-1- or HIV-2-infected T cells. It is interesting to note that HIV protease degrades human bcl2 [84] and that HIV Nef, an HIV accessory' protein, induces apoptosis [85]. Thus this mechanism of HIV induction of apoptosis breaks the typical paradigm where viruses encode proteins that inhibit apoptosis (see Table 1).

HIV encodes several other "accessory" proteins that are essential for HIV infectivity and pathogenesis in vivo. These proteins antagonize the innate immune response in several ways. Nef mediates activation of MAPK signaling to AP-1, which is suggested to activate viral replication [86, 87]. HIV Vif and Vpr degrade IRF3, thereby inhibiting signaling to IRF3 and interferon production [88]. Thus, although inhibition of adaptive immunity by HIV is well known, innate immune evasion plays an important role in HIV pathogenesis.

\section{Conclusion: Innate Immunoevasion-From Insight to Innovation}

Many viral mechanisms have evolved to evade the immune response. Surprisingly, the general outline of innate antiviral mechanism is remarkably persistent throughout evolution, such as DNA restriction/dicing and programmed cell death; however, differences between innate immune responses of distinct organisms are often more striking and may hint at novel innate immune evasion pathways still undiscovered in mammalian virus-host interactions. Signaling to interferon resembles antiviral protein induction in Drosophila and in some respects, even in bacteria. The evolutionary conservation of these mechanisms suggests their study will advance understanding of viral pathogenesis and that these pathways would be worthy targets of antiviral inhibitors.

In this regard, there are several promising antiviral therapies targeting viral innate immunoevasion genes. $\mathrm{HCV}$ protease inhibitors have been suggested to inhibit $\mathrm{HCV}$ innate immunoevasion, presumably by preventing MAVS digestion $[38,89]$, and thereby permitting critical signaling to interferon. An in vitro study of the N1 vaccinia virus virulence factor and innate immune evasion protein identified chemical inhibitors of its antiapoptotic function [90]. This is surprising as $\mathrm{N} 1$ does not mediate cell death in vitro, where these inhibitors were tested [91, 92]. These findings highlight the difficulty of studying certain innate immune inhibitors in vitro. Nevertheless, targeting potent virulence factors of viral pathogens represent a promising and entirely new approach to antiviral drug design-beyond drugs that exclusively target viral enzymes responsible for replication. Perhaps the most promising application of these studies is in the development of highly immunogenic live vaccines that contain deletions of innate immune evasion genes outlined here. Such vaccine would have the potential to be safer and potentially more immunogenic vaccine viruses by virtue of their attenuated ability to mediate innate immune suppression.

\section{Abbreviations}

PAMP: Pathogen-associated molecular pattern

NLR: Nucleotide oligomerization domain-Like receptor

TLR: Toll-like receptor

RLR: RIG-I-like receptor

RIG-I: Retinoic acid-inducible gene-I

MDA-5: Melanoma differentiation-associated gene-5

CARD: Caspase activation and recruitment domain

MAVS: Mitochondrial antiviral signaling protein

LPS: Lipopolysaccharide

TIR: $\quad$ Toll/IL-1 interacting receptor

NLRP3: Nucleotide oligomerization domain-like receptor Protein 3

ASC: $\quad$ Apoptosis-associated speck-like protein containing caspase recruitment domain

DAI: DNA-dependent activator of interferon

STING: Stimulator of interferon genes

CRISPR: Clustered regularly sPaced short palindromic repeats

Psp: $\quad$ Phage shock protein

VACV: Vaccinia virus

EBV: $\quad$ Epstein-Barr virus

KSHV: Kaposi'ssarcoma herpesvirus 


$\begin{array}{ll}\text { HCV: } & \begin{array}{l}\text { Hepatitis C virus } \\ \text { NPH-I: }\end{array} \\ & \begin{array}{l}\text { Poxvirus nucleoside triphosphate } \\ \text { phosphohydrolase I }\end{array} \\ \text { IFN: } & \text { Interferon } \\ \text { NS1: } & \text { Nonstructural protein } 1 \\ \text { DDX3: } & \text { DEAD/H Box } 3 \\ \text { IKK: } & \text { Inhibitor ofkappa-B kinase } \\ \text { TBK: } & \text { TANK-binding kinase 1 } \\ \text { IRAK: } & \text { IL-1 receptor-associated kinase-1 } \\ \text { IRF3: } & \text { Interferon response factor 3 } \\ \text { IL: } & \text { Interleukin } \\ \text { AIM-2: } & \text { Absent in melanoma-2 }\end{array}$

TRIM 25: Tripartite motif-containing protein 25.

\section{Acknowledgments}

The authors would like to acknowledge the critical reading of the paper by Neal Silverman. This project has been funded in whole or in part with funds from the National Institute of Allergy and Infectious Diseases, National Institutes of Health: R01 AI070940 to WLM.

\section{References}

[1] C. A. Janeway Jr., "Approaching the asymptote? Evolution and revolution in immunology," Cold Spring Harbor Symposia on Quantitative Biology, vol. 54, no. 1, pp. 1-13, 1989.

[2] M. Yoneyama, M. Kikuchi, T. Natsukawa et al., "The RNA helicase RIG-I has an essential function in double-stranded RNA-induced innate antiviral responses," Nature Immunology, vol. 5, no. 7, pp. 730-737, 2004.

[3] T. Kawai, K. Takahashi, S. Sato et al., "IPS-1, an adaptor triggering RIG-I- and Mda5-mediated type I interferon induction," Nature Immunology, vol. 6, no. 10, pp. 981-988, 2005.

[4] M. Yoneyama, M. Kikuchi, K. Matsumoto et al., "Shared and unique functions of the DExD/H-box helicases RIG-I, MDA5, and LGP2 in antiviral innate immunity," Journal of Immunology, vol. 175, no. 5, pp. 2851-2858, 2005.

[5] A. P. Kalverda, G. S. Thompson, A. Vogel et al., "Poxvirus $\mathrm{K} 7$ protein adopts a Bcl-2 fold: biochemical mapping of its interactions with human DEAD box RNA helicase DDX3," Journal of Molecular Biology, vol. 385, no. 3, pp. 843-853, 2009.

[6] N. K. Tanner and P. Linder, "DExD/H box RNA helicases: from generic motors to specific dissociation functions," Molecular Cell, vol. 8, no. 2, pp. 251-262, 2001.

[7] S. Rothenfusser, N. Goutagny, G. DiPerna et al., "The RNA helicase Lgp2 inhibits TLR-independent sensing of viral replication by retinoic acid-inducible gene-I," Journal of Immunology, vol. 175, no. 8, pp. 5260-5268, 2005.

[8] T. Satoh, H. Kato, Y. Kumagai et al., "LGP2 is a positive regulator of RIG-I- and MDA5-mediated antiviral responses," Proceedings of the National Academy of Sciences of the United States of America, vol. 107, no. 4, pp. 1512-1517, 2010.

[9] R. B. Seth, L. Sun, C. K. Ea, and Z. J. Chen, "Identification and characterization of MAVS, a mitochondrial antiviral signaling protein that activates NF- $\kappa$ B and IRF3," Cell, vol. 122, no. 5, pp. 669-682, 2005.

[10] Q. Sun, L. Sun, H. H. Liu et al., "The specific and essential role of MAVS in antiviral innate immune responses," Immunity, vol. 24, no. 5, pp. 633-642, 2006.
[11] S. Akira, "Mammalian Toll-like receptors," Current Opinion in Immunology, vol. 15, no. 2, p. 238, 2003.

[12] F. Heil, H. Hemmi, H. Hochrein et al., "Species-specific recognition of single-stranded RNA via Till-like receptor 7 and 8," Science, vol. 303, no. 5663, pp. 1526-1529, 2004.

[13] S. Sharma, B. R. TenOever, N. Grandvaux, G. P. Zhou, R. Lin, and J. Hiscott, "Triggering the interferon antiviral response through an IKK-related pathway," Science, vol. 300, no. 5622, pp. 1148-1151, 2003.

[14] N. Silverman and T. Maniatis, "NF- $\kappa$ B signaling pathways in mammalian and insect innate immunity," Genes and Development, vol. 15, no. 18, pp. 2321-2342, 2001.

[15] S. M. McWhirter, K. A. Fitzgerald, J. Rosains, D. C. Rowe, D. T. Golenbock, and T. Maniatis, "IFN-regulatory factor 3-dependent gene expression is defective in Tbk1-deficient mouse embryonic fibroblasts," Proceedings of the National Academy of Sciences of the United States of America, vol. 101, no. 1, pp. 233-238, 2004.

[16] S. E. Doyle, S. A. Vaidya, R. O'Connell et al., "IRF3 mediates a TLR3/TLR4-specific antiviral gene program," Immunity, vol. 17, no. 3, pp. 251-263, 2002.

[17] G. Gautier, M. Humbert, F. Deauvieau et al., "A type I interferon autocrine-paracrine loop is involved in Toll-like receptor-induced interleukin- 12 p70 secretion by dendritic cells," Journal of Experimental Medicine, vol. 201, no. 9, pp. 1435-1446, 2005.

[18] M. M. Whitmore, M. J. DeVeer, A. Edling et al., "Synergistic activation of innate immunity by double-stranded RNA and CpG DNA promotes eahanced antitumor activity," Cancer Research, vol. 64, no. 16, pp. 5850-5860, 2004.

[19] K. Hoebe, X. Du, P. Georgel et al., "Identification of Lps2 as a key transducer of MyD88-independent TIR signalling," Nature, vol. 424, no. 6950, pp. 743-748, 2003.

[20] L. Franchi, T. Eigenbrod, R. Muñoz-Planillo, and G. Nuñez, "The inflammasome: a caspase-1-activation platform that regulates immune responses and disease pathogenesis," Nature Immunology, vol. 10, no. 3, pp. 241-247, 2009.

[21] V. Hornung, A. Ablasser, M. Charrel-Dennis et al., "AIM2 recognizes cytosolic dsDNA and forms a caspase-1-activating inflammasome with ASC," Nature, vol. 458, no. 7237, pp. 514$518,2009$.

[22] M. Lamkanfi and V. M. Dixit, "Inflammasomes: guardians of cytosolic sanctity," Immunological Reviews, vol. 227, no. 1, pp. 95-105, 2009.

[23] K. J. Ishii, C. Coban, H. Kato et al., "A toll-like receptorindependent antiviral response induced by double-stranded B-form DNA," Nature Immunology, vol. 7, no. 1, pp. 40-48, 2006.

[24] D. B. Stetson and R. Medzhitov, "Recognition of cytosolic DNA activates an IRF3-dependent innate immune response," Immunity, vol. 24, no. 1, pp. 93-103, 2006.

[25] Z. Wang, M. K. Choi, T. Ban et al., "Regulation of innate immune responses by DAI (DLM-1/ZBP1) and other DNAsensing molecules," Proceedings of the National Academy of Sciences of the United States of America, vol. 105, no. 14, pp. 5477-5482, 2008.

[26] D. Bikard and L. A. Marraffini, "Innate and adaptive immunity in bacteria: mechanisms of programmed genetic variation to fight bacteriophages," Current Opinion in Immunology, vol. 24, no. 1, pp. 15-20, 2012.

[27] W. C. Cheng and J. M. Hardwick, "A quorum on bacterial programmed cell death,” Molecular Cell, vol. 28, no. 4, pp. 515-517, 2007. 
[28] Y.-H. Han, Y.-J. Luo, Q. Wu et al., "RNA-based immunity terminates viral infection in adult Drosophila in the absence of viral suppression of RNA interference: characterization of viral small interfering RNA populations in wild-type and mutant flies," Journal of Virology, vol. 85, no. 24, pp. 13153-13163, 2011.

[29] J. H. Wang, S. Valanne, and M. Ramet, "Drosophila as a model for antiviral immunity," World Journal of Biological Chemistry, vol. 1, no. 5, pp. 151-159, 2010.

[30] D. Galiana-Arnoux, C. Dostert, A. Schneemann, J. A. Hoffmann, and J. L. Imler, "Essential function in vivo for Dicer2 in host defense against RNA viruses in drosophila," Nature Immunology, vol. 7, no. 6, pp. 590-597, 2006.

[31] N. E. Crook, R. J. Clem, and L. K. Miller, "An apoptosisinhibiting baculovirus gene with a zinc finger-like motif," Journal of Virology, vol. 67, no. 4, pp. 2168-2174, 1993.

[32] B. A. Hay, T. Wolff, and G. M. Rubin, "Expression of baculovirus P35 prevents cell death in Drosophila," Development, vol. 120, no. 8, pp. 2121-2129, 1994.

[33] R. L. Kuo, C. Zhao, M. Malur, and R. M. Krug, "Influenza A virus strains that circulate in humans differ in the ability of their NS1 proteins to block the activation of IRF3 and interferon- $\beta$ transcription," Virology, vol. 408, no. 2, pp. 146 $158,2010$.

[34] J. O. Langland and B. L. Jacobs, "The role of the PKRinhibitory genes, $\mathrm{E} 3 \mathrm{~L}$ and $\mathrm{K} 3 \mathrm{~L}$, in determining vaccinia virus host range," Virology, vol. 299, no. 1, pp. 133-141, 2002.

[35] G. DiPerna, J. Stack, A. G. Bowie et al., "Poxvirus protein N1L targets the I- $\kappa \mathrm{B}$ kinase complex, inhibits signaling to NF- $\kappa \mathrm{B}$ by the tumor necrosis factor superfamily of receptors, and inhibits NF- $\kappa \mathrm{B}$ and IRF3 signaling by toll-like receptors," The Journal of Biological Chemistry, vol. 279, no. 35, pp. 3657036578, 2004.

[36] A. Bowie, E. Kiss-Toth, J. A. Symons, G. L. Smith, S. K. Dower, and L. A. J. O'Neill, "A46R and A52R from vaccinia virus are antagonists of host IL-1 and toll-like receptor signaling," Proceedings of the National Academy of Sciences of the United States of America, vol. 97, no. 18, pp. 10162-10167, 2000.

[37] J. M. Gonzlez and M. Esteban, "A poxvirus Bcl-2-like gene family involved in regulation of host immune response: sequence similarity and evolutionary history," Virology Journal, vol. 7, article 59, 2010.

[38] C. L. Johnson, D. M. Owen, and M. Gale Jr., "Functional and therapeutic analysis of hepatitis $\mathrm{C}$ virus NS3.4A protease control of antiviral immune defense," The Journal of Biological Chemistry, vol. 282, no. 14, pp. 10792-10803, 2007.

[39] A. Hosui, K. Ohkawa, H. Ishida et al., "Hepatitis C virus core protein differently regulates the JAK-STAT signaling pathway under interleukin-6 and interferon- $\gamma$ stimuli," The Journal of Biological Chemistry, vol. 278, no. 31, pp. 28562-28571, 2003.

[40] M. J. Gale Jr., M. J. Korth, and M. G. Katze, "Repression of the PKR protein kinase by the hepatitis C virus NS5A protein: a potential mechanism of interferon resistance," Clinical and Diagnostic Virology, vol. 10, no. 2-3, pp. 157-162, 1998.

[41] B. Moss, "Poxviridae: the viruses and their replication," in Fields Virology, D. M. Knipe and P. M. Howley, Eds., vol. 2, pp. 2849-2883, Lippincott, Williams \& Wilkins, Philadelphia, Pa, USA, 2001.

[42] K. Li, E. Foy, J. C. Ferreon et al., "Immune evasion by hepatitis $\mathrm{C}$ virus NS3/4A protease-mediated cleavage of the Toll-like receptor 3 adaptor protein TRIF," Proceedings of the National Academy of Sciences of the United States of America, vol. 102, no. 8, pp. 2992-2997, 2005.
[43] J. P. Parisien, D. Bamming, A. Komuro et al., "A shared interface mediates Paramyxovirus interference with antiviral RNA helicases MDA5 and LGP2," Journal of Virology, vol. 83, no. 14, pp. 7252-7260, 2009.

[44] S. M. Gregory, B. K. Davis, J. A. West et al., "Discovery of a viral NLR homolog that inhibits the inflammasome," Science, vol. 331, no. 6015, pp. 330-334, 2011.

[45] M. M. Rahman and G. McFadden, "Myxoma virus lacking the pyrin-like protein M013 is sensed in human myeloid cells by both NLRP3 and multiple toll-like receptors, which independently activate the inflammasome and NF- $\kappa \mathrm{B}$ innate response pathways," Journal of Virology, vol. 85, no. 23, pp. 12505-12517, 2011.

[46] M. Thome, P. Schneider, K. Hofmann et al., "Viral FLICEinhibitory proteins (FLIPs) prevent apoptosis induced by death receptors," Nature, vol. 386, no. 6624, pp. 517-521, 1997.

[47] S. Henderson, D. Huen, M. Rowe, C. Dawson, G. Johnson, and A. Rickinson, "Epstein-Barr virus-coded BHRF1 protein, a viral homologue of $\mathrm{Bcl}-2$, protects human $\mathrm{B}$ cells from programmed cell death," Proceedings of the National Academy of Sciences of the United States of America, vol. 90, no. 18, pp. 8479-8483, 1993.

[48] W. L. Marshall, C. Yim, E. Gustafson et al., "Epstein-Barr virus encodes a novel homolog of the bcl-2 oncogene that inhibits apoptosis and associates with Bax and Bak," Journal of Virology, vol. 73, no. 6, pp. 5181-5185, 1999.

[49] R. Sarid, T. Sato, R. A. Bohenzky, J. J. Russo, and Y. Chang, "Kaposi's sarcoma-associated herpesvirus encodes a functional Bcl-2 homologue," Nature Medicine, vol. 3, no. 3, pp. 293-298, 1997.

[50] T. W. Sedlak, Z. N. Oltvai, E. Yang et al., "Multiple Bcl2 family members demonstrate selective dimerizations with Bax," Proceedings of the National Academy of Sciences of the United States of America, vol. 92, no. 17, pp. 7834-7838, 1995.

[51] D. C. S. Huang, J. M. Adams, and S. Cory, "The conserved $\mathrm{N}$-terminal $\mathrm{BH} 4$ domain of Bcl-2 homologues is essential for inhibition of apoptosis and interaction with CED-4," EMBO Journal, vol. 17, no. 4, pp. 1029-1039, 1998.

[52] A. Gross, J. M. McDonnell, and S. J. Korsmeyer, "BCL-2 family members and the mitochondria in apoptosis," Genes and Development, vol. 13, no. 15, pp. 1899-1911, 1999.

[53] M. Mandal, S. B. Maggirwar, N. Sharma, S. H. Kaufmann, S. C. Sun, and R. Kumar, "Bcl-2 prevents CD95 (Fas/APO1)-induced degradation of lamin $\mathrm{B}$ and poly(ADP-ribose) polymerase and restores the NF- $\kappa \mathrm{B}$ signaling pathway," The Journal of Biological Chemistry, vol. 271, no. 48, pp. 3035430359, 1996.

[54] L. A. Kirshenbaum, "Bcl-2 intersects the NFkB signalling pathway and suppresses apoptosis in ventricular myocytes," Clinical and Investigative Medicine, vol. 23, no. 5, pp. 322-330, 2000.

[55] K. M. Regula, K. Ens, and L. A. Kirshenbaum, "IKK $\beta$ is required for Bcl-2-mediated NF- $\kappa \mathrm{B}$ activation in ventricular myocytes," The Journal of Biological Chemistry, vol. 277, no. 41, pp. 38676-38682, 2002.

[56] Q. Li, G. Estepa, S. Memet, A. Israel, and I. M. Verma, "Complete lack of NF- $\kappa$ B activity in IKK1 and IKK2 doubledeficient mice: additional defect in neurulation," Genes and Development, vol. 14, no. 14, pp. 1729-1733, 2000.

[57] M. Karin and Y. Ben-Neriah, "Phosphorylation meets ubiquitination: the control of NF- $\kappa \mathrm{B}$ activity," Annual Review of Immunology, vol. 18, pp. 621-663, 2000. 
[58] J. V. Hartig and K. Förstemann, "Loqs-PD and R2D2 define independent pathways for RISC generation in Drosophila," Nucleic Acids Research, vol. 39, no. 9, pp. 3836-3851, 2011.

[59] M. Hussain, R. J. Taft, and S. Asgari, "An insect virus-encoded microrna regulates viral replication," Journal of Virology, vol. 82, no. 18, pp. 9164-9170, 2008.

[60] G. Jovanovic, C. Engl, and M. Buck, "Physical, functional and conditional interactions between $\mathrm{ArcAB}$ and phage shock proteins upon secretin-induced stress in Escherichia coli," Molecular Microbiology, vol. 74, no. 1, pp. 16-28, 2009.

[61] R. A. Zambon, M. Nandakumar, V. W. Vakharia, and L. P. Wu, "The Toll pathway is important for an antiviral response in Drosophila," Proceedings of the National Academy of Sciences of the United States of America, vol. 102, no. 20, pp. 7257-7262, 2005.

[62] S. Deddouche, N. Matt, A. Budd et al., "The DExD/H-box helicase Dicer-2 mediates the induction of antiviral activity in drosophila," Nature Immunology, vol. 9, no. 12, pp. 1425-1432, 2008.

[63] M. E. Grether, J. M. Abrams, J. Agapite, K. White, and H. Steller, "The head involution defective gene of Drosophila melanogaster functions in programmed cell death," Genes and Development, vol. 9, no. 14, pp. 1694-1708, 1995.

[64] E. C. LaCasse, S. Baird, R. G. Korneluk, and A. E. MacKenzie, "The inhibitors of apoptosis (IAPs) and their emerging role in cancer," Oncogene, vol. 17, no. 25, pp. 3247-3259, 1998.

[65] A. Alcamí, J. A. Symons, and G. L. Smith, "The vaccinia virus soluble alpha/beta interferon (IFN) receptor binds to the cell surface and protects cells from the antiviral effects of IFN," Journal of Virology, vol. 74, no. 23, pp. 11230-11239, 2000.

[66] B. Billings, S. A. Smith, Z. Zhang, D. K. Lahiri, and G. J. Kotwal, "Lack of N1L gene expression results in a significant decrease of vaccinia virus replication in mouse brain," Annals of the New York Academy of Sciences, vol. 1030, pp. 297-302, 2004.

[67] Z. Zhang, M. R. Abrahams, L. A. Hunt et al., "The vaccinia virus N1L protein influences cytokine secretion in vitro after infection," Annals of the New York Academy of Sciences, vol. 1056, pp. 69-86, 2005.

[68] M. T. Harte, I. R. Haga, G. Maloney et al., "The poxvirus protein A52R targets toll-like receptor signaling complexes to suppress host defense," Journal of Experimental Medicine, vol. 197, no. 3, pp. 343-351, 2003.

[69] C. A. Ray, R. A. Black, S. R. Kronheim et al., "Viral inhibition of inflammation: cowpox virus encodes an inhibitor of the interleukin- $1 \beta$ converting enzyme," Cell, vol. 69, no. 4, pp. 597-604, 1992.

[70] R. Mills, M. Rozanov, A. Lomsadze, T. Tatusova, and M. Borodovsky, "Improving gene annotation of complete viral genomes," Nucleic Acids Research, vol. 31, no. 23, pp. 70417055, 2003.

[71] Q. Huang, A. M. Petros, H. W. Virgin, S. W. Fesik, and E. T. Olejniczak, "Solution structure of the BHRF1 protein from Epstein-Barr virus, a homolog of human Bcl-2," Journal of Molecular Biology, vol. 332, no. 5, pp. 1123-1130, 2003.

[72] M. Altmann and W. Hammerschmidt, "Epstein-Barr virus provides a new paradigm: a requirement for the immediate inhibition of apoptosis," PLoS Biology, vol. 3, no. 12, article e404, 2005.

[73] N. J. Bump, M. Hackett, M. Hugunin et al., "Inhibition of ICE family proteases by baculovirus antiapoptotic protein p35," Science, vol. 269, no. 5232, pp. 1885-1888, 1995.
[74] E. Meinl, H. Fickenscher, M. Thome, J. Tschopp, and B. Fleckenstein, "Anti-apoptotic strategies of lymphotropic viruses," Immunology Today, vol. 19, no. 10, pp. 474-479, 1998.

[75] T. Kondo, T. Yokokura, and S. Nagata, "Activation of distinct caspase-like proteases by Fas and reaper in Drosophila cells," Proceedings of the National Academy of Sciences of the United States of America, vol. 94, no. 22, pp. 11951-11956, 1997.

[76] N. Field, W. Low, M. Daniels et al., "KSHV vFLIP binds to IKK- $\gamma$ to activate IKK," Journal of Cell Science, vol. 116, no. 18, pp. 3721-3728, 2003.

[77] M. Diaz-Guerra, J. S. Kahn, and M. Esteban, "A mutation of the nucleoside triphosphate phosphohydrolase I (NPHI) gene confers sensitivity of vaccinia virus to interferon," Virology, vol. 197, no. 1, pp. 485-491, 1993.

[78] A. J. V. Thompson and J. G. McHutchison, "Antiviral resistance and specifically targeted therapy for HCV (STAT-C)," Journal of Viral Hepatitis, vol. 16, no. 6, pp. 377-387, 2009.

[79] H. Chung, T. Watanabe, M. Kudo, and T. Chiba, "Hepatitis C virus core protein induces homotolerance and cross-tolerance to Toll-like receptor ligands by activation of Toll-like receptor 2," Journal of Infectious Diseases, vol. 202, no. 6, pp. 853-861, 2010.

[80] W. Lin, W. H. Choe, Y. Hiasa et al., "Hepatitis C virus expression suppresses interferon signaling by degrading STAT1," Gastroenterology, vol. 128, no. 4, pp. 1034-1041, 2005.

[81] A. L. Hartman, J. S. Towner, and S. T. Nichol, "A C-terminal basic amino acid motif of Zaire ebolavirus VP35 is essential for type I interferon antagonism and displays high identity with the RNA-binding domain of another interferon antagonist, the NS1 protein of influenza a virus," Virology, vol. 328, no. 2, pp. 177-184, 2004.

[82] A. Pichlmair, O. Schulz, C. P. Tan et al., "RIG-I-mediated antiviral responses to single-stranded RNA bearing $5^{\prime}$ phosphates," Science, vol. 314, no. 5801, pp. 997-1001, 2006.

[83] Y. Watanabe, N. Ohtaki, Y. Hayashi, K. Ikuta, and K. Tomonaga, "Autogenous translational regulation of the borna disease virus negative control factor $\mathrm{X}$ from polycistronic mRNA using host RNA helicases," PLoS Pathogens, vol. 5, no. 11, Article ID e1000654, 2009.

[84] P. R. Strack, M. W. Frey, C. J. Rizzo et al., "Apoptosis mediated by HIV protease is preceded by cleavage of bcl-2," Proceedings of the National Academy of Sciences of the United States of America, vol. 93, no. 18, pp. 9571-9576, 1996.

[85] A. Rasola, D. Gramaglia, C. Boccaccio, and P. M. Comoglio, "Apoptosis enhancement by the HIV-1 Nef protein," Journal of Immunology, vol. 166, no. 1, pp. 81-88, 2001.

[86] L. Tuosto, B. Marinari, M. Andreotti, M. Federico, and E. Piccolella, "Vav exchange factor counteracts the HIV-1 Nefmediated decrease of plasma membrane GM1 and NF-AT activity in T cells," European Journal of Immunology, vol. 33, no. 8, pp. 2186-2196, 2003.

[87] A. Varin, S. K. Manna, V. Quivy et al., "Exogenous Nef protein activates NF- $\kappa \mathrm{B}, \mathrm{AP}-1$, and $\mathrm{c}$-Jun N-terminal kinase and stimulates HIV transcription in promonocytic cells: role in AIDS pathogenesis," The Journal of Biological Chemistry, vol. 278, no. 4, pp. 2219-2227, 2003.

[88] A. Okumura, T. Alce, B. Lubyova, H. Ezelle, K. Strebel, and P. M. Pitha, "HIV-1 accessory proteins VPR and Vif modulate antiviral response by targeting IRF-3 for degradation," Virology, vol. 373, no. 1, pp. 85-97, 2008.

[89] J. A. Thomson and R. B. Perni, "Hepatitis C virus NS3.4A protease inhibitors: countering viral subversion in vitro and 
showing promise in the clinic," Current Opinion in Drug Discovery and Development, vol. 9, no. 5, pp. 606-617, 2006.

[90] N. Bartlett, J. A. Symons, D. C. Tscharke, and G. L. Smith, “The vaccinia virus N1L protein is an intracellular homodimer that promotes virulence," Journal of General Virology, vol. 83, no. 8, pp. 1965-1976, 2002.

[91] G. J. Kotwal, A. W. Hugin, and B. Moss, "Mapping and insertional mutagenesis of a vaccinia virus gene encoding a 13,800-Da secreted protein," Virology, vol. 171, no. 2, pp. 579$587,1989$.

[92] A. V. Cheltsov, M. Aoyagi, A. Aleshin et al., "Vaccinia virus virulence factor N1L is a novel promising target for antiviral therapeutic intervention," Journal of Medicinal Chemistry, vol. 53, no. 10, pp. 3899-3906, 2010. 

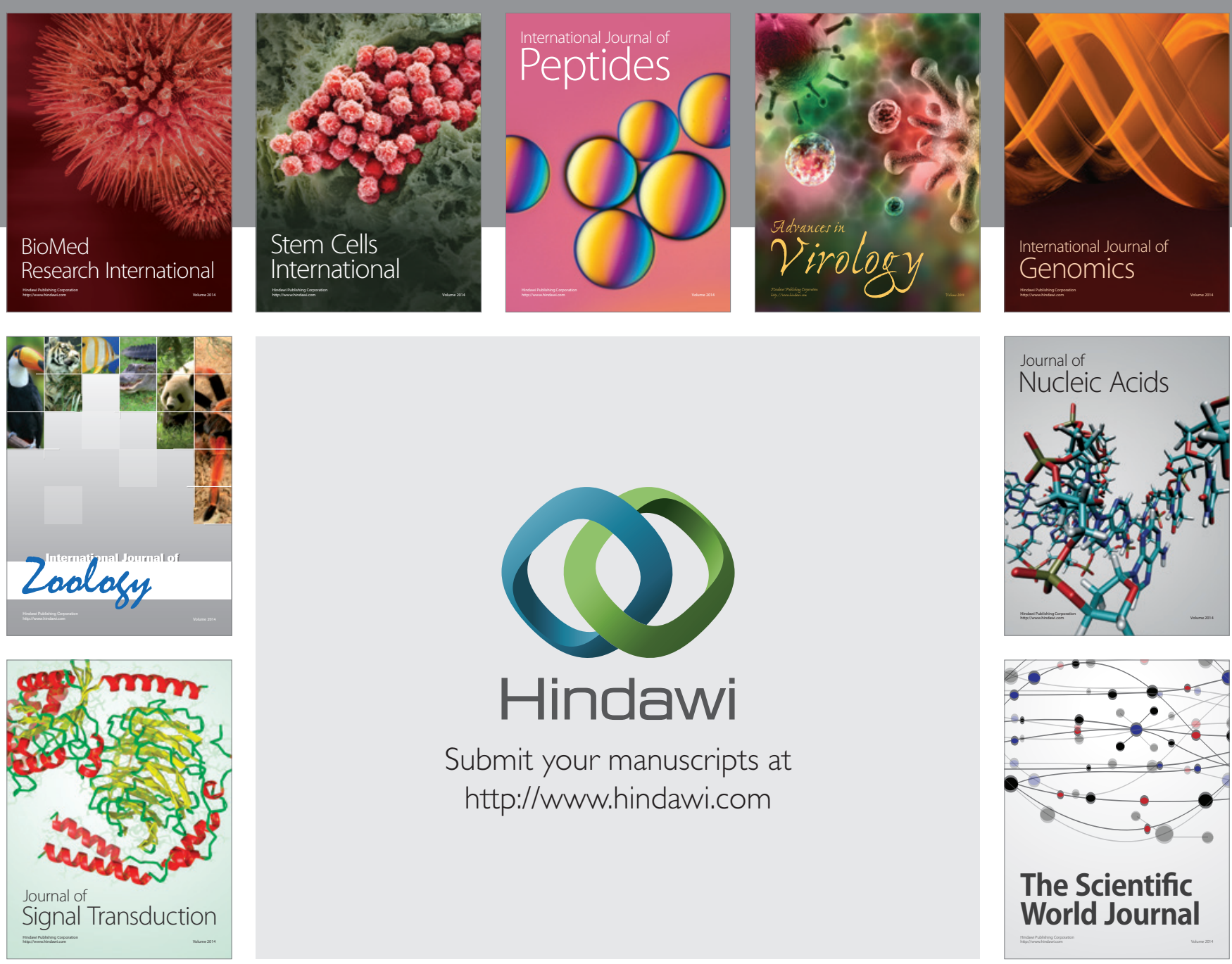

Submit your manuscripts at

http://www.hindawi.com
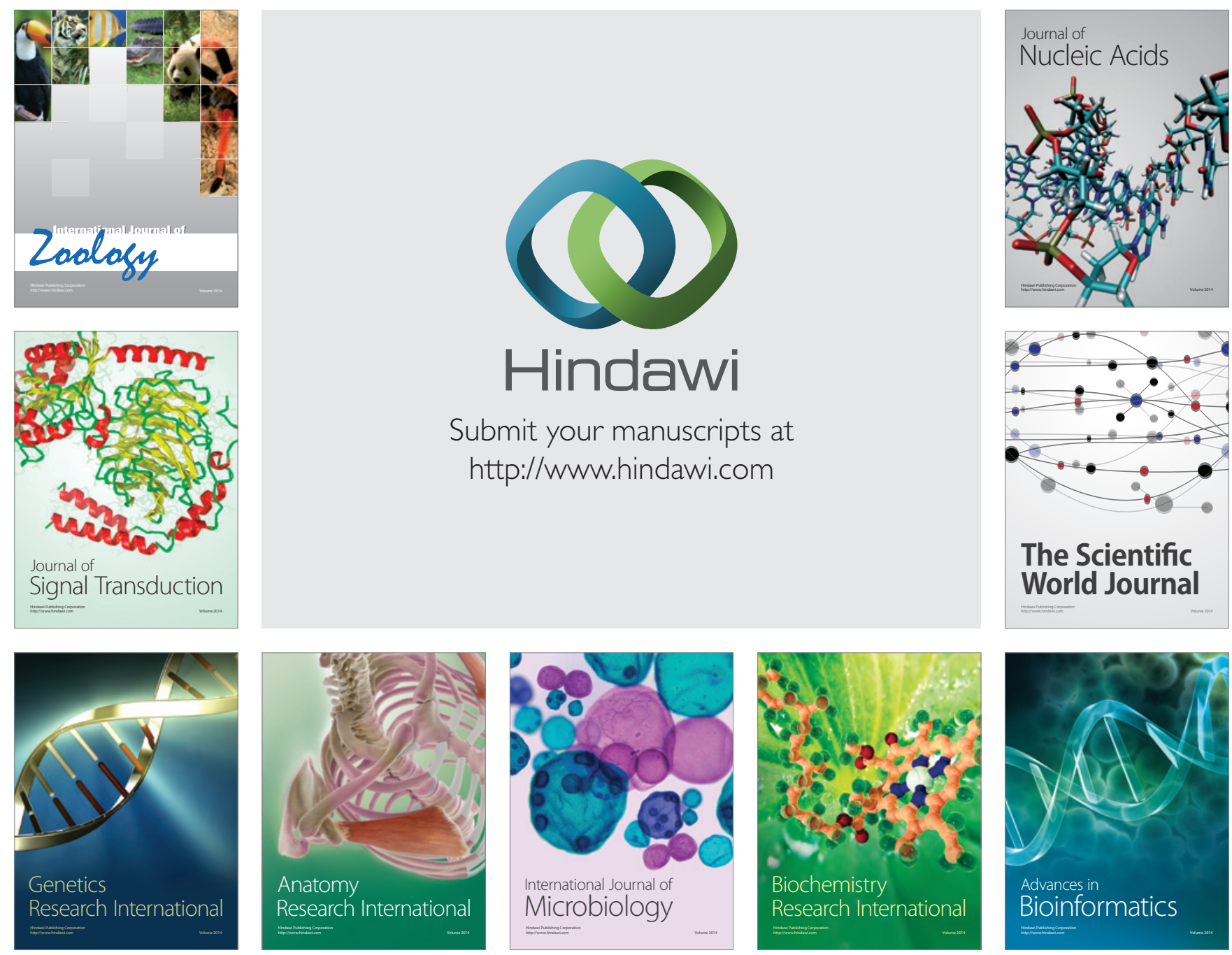

The Scientific World Journal
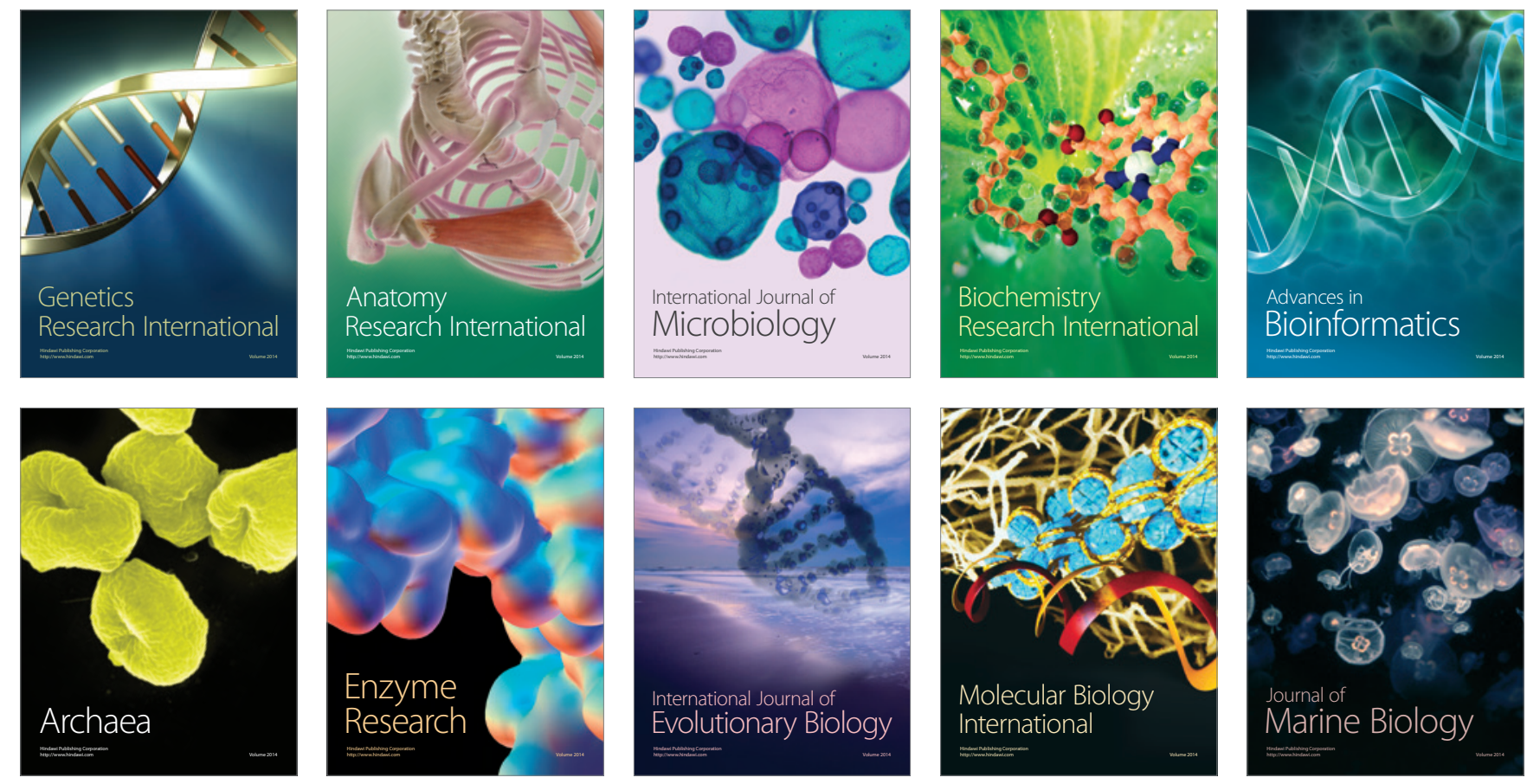